\title{
Ground-state phase diagram of the frustrated spin-1/2 two-leg honeycomb ladder
}

\author{
Qiang Luo, ${ }^{1}$ Shijie Hu, ${ }^{2}$, Jize Zhao,${ }^{3}$ Alexandros Metavitsiadis, ${ }^{2,4}$ Sebastian Eggert, ${ }^{2}$ and Xiaoqun Wang ${ }^{5,6}$, 而 \\ ${ }^{1}$ Department of Physics, Renmin University of China, Beijing 100872, China \\ ${ }^{2}$ Department of Physics and Research Center Optimas, \\ Technische Universität Kaiserslautern, 67663 Kaiserslautern, Germany \\ ${ }^{3}$ Center for Interdisciplinary Studies, Lanzhou University, Lanzhou 730000, China \\ ${ }^{4}$ Institute for Theoretical Physics, Technical University Braunschweig, 38106 Braunschweig, Germany \\ ${ }^{5}$ Key Laboratory of Artificial Structures and Quantum Control (Ministry of Education), \\ School of Physics and Astronomy, Tsung-Dao Lee Institute, \\ Shanghai Jiao Tong University, Shanghai 200240, China \\ ${ }^{6}$ Collaborative Innovation Center for Advanced Microstructures, Nanjing 210093, China
}

(Dated: April 3, 2018)

\begin{abstract}
We investigate a spin-1/2 two-leg honeycomb ladder with frustrating next-nearest-neighbor (NNN) coupling along the legs, which is equivalent to two $J_{1^{-}} J_{2}$ spin chains coupled with $J_{\perp}$ at odd rungs. The full parameter region of the model is systematically studied using conventional and infinite density-matrix renormalization group as well as bosonization. The rich phase diagram consists of five distinct phases: a Haldane phase, a NNN-Haldane phase and a staggered dimer phase when $J_{\perp}<0$; a rung singlet phase and a columnar dimer phase when $J_{\perp}>0$. An interesting reentrant behavior from the dimerized phase into the Haldane phase is found as the frustration $J_{2}$ increases. The universalities of the critical phase transitions are fully analyzed. Phase transitions between dimerized and disordered phases belong to the two-dimensional Ising class with central charge $c=1 / 2$. The transition from the Haldane phase to NNN-Haldane phase is of a weak topological first order, while the continuous transition between the Haldane phase and rung singlet phase has central charge $c=2$.
\end{abstract}

\section{INTRODUCTION}

Frustration due to competing interactions in quantum spin systems often gives rise to interesting and rich quantum phase diagrams $\stackrel{1,2}{\frac{12}{2}}$ The prototypical example of a spin- $1 / 2$ chain with frustrating next-nearestneighbor (NNN) interactions $J_{2}$ is known to undergo a Berezinskii-Kosteriz-Thouless (BKT) transition from a gapless Tomonaga-Luttinger liquid (TLL) phase to a gapped dimerized phase with spontaneously broken translational symmetry $\underline{\underline{3}} \underline{\underline{7}}$ at $J_{2, c} \simeq 0.241167 J_{1}$ where $J_{1}$ is the nearest neighbor (NN) interaction along the chain. At the Majumdar-Ghosh point $\underline{\underline{8} .9} J_{2}=J_{1} / 2$ the ground state is known exactly and for $J_{2}>J_{1} / 2$ incommensurate spiral spin-spin correlations emerge $\underline{7,10}$ For comparison the spin- 1 chain is in a gapped Haldane state $\frac{11,12}{12}$ with topological string order ${ }^{13,14}$ for small $J_{2}$, but the correlations become incommensurate for $J_{2} \gtrsim$ $0.284 J_{1}$ and a transition to a NNN-Haldane phase occurs at $J_{2, c} \simeq 0.7444 J_{1}$, where valance bonds link NNN sites,$\frac{15-22}{2}$ which is sometimes also referred to as a double Haldane phase.

Quantum spin ladders have been intensively studied in order to systematically extend the systems towards twodimensions (2D) $\stackrel{23}{-41}$ For unfrustrated two-leg spin-1/2 ladders the ground state is either in a rung singlet (RS) phase or in a Haldane phase for positive or negative rung couplings $J_{\perp}$, respectively. By including frustrating couplings between the chains $J_{\times}$the possibility of a columnar dimerized (CD) phase between the Haldane and RS phases was proposed, $\stackrel{29,42,43}{\text { but the numerical }}$ evidence remains controversial for this case $30-33$ On the

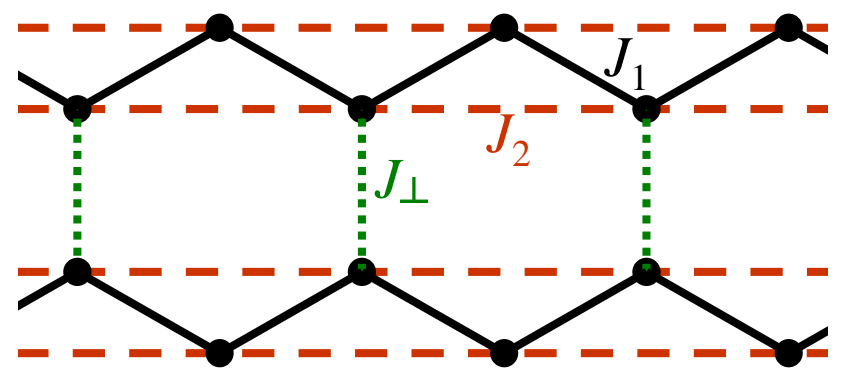

FIG. 1. Illustration of interactions on a frustrated two-leg spin-1/2 honeycomb ladder.

other hand, small frustrating NNN couplings $J_{2}$ on the legs are known to induce and stabilize dimerized phases in the columnar or the staggered order, $\underline{\underline{34}} \underline{\underline{41}}$ and in some cases reentrant behavior for larger $J_{2}$ may occur $\underline{\underline{36}}$

We now analyze the honeycomb ladder as shown in Fig. 1 consisting of two spin- $1 / 2$ legs with a rungalternating coupling. $\stackrel{44}{ }$ In this paper we focus on the effect of frustrating NNN couplings on the legs $\left(J_{2}\right)$, while frustrating inter-chain couplings $\left(J_{\times}\right)$may be considered in a later work. As a 2D model the frustrated honeycomb model has received considerable attention also in the context of longer range interactions and spinliquid physics $\stackrel{45-49}{-4 x p e r i m e n t a l l y, ~ t h e ~ h o n e y c o m b ~ l a d-~}$ der has recently been realized in a four azide copper coordination compound $\left[\mathrm{Cu}_{2} \mathrm{~L}^{1}\left(\mathrm{~N}_{3}\right)_{4}\right]_{n}\left(\mathrm{~L}^{1}=2\right.$, 6-bis (4, 5-dihydrooxazol-2-yl) pyridine), with antiferromagnetic intrachain coupling and ferromagnetic inter-chain coupling .50 Using optical lattices a honeycomb structure can also be constructed for interacting ultrcold gases $\underline{51}$ 
The paper is organized as follows: In Sec. II] we introduce the model Hamiltonian and discuss the symmetries. The phase diagram from the numerical simulations is summarized and discussed in Sec.[II] In Sec.[IV we use bosonization and the renormalization group (RG) to obtain an analytic approach to predict the transition lines and compare with numerics before a detailed analysis of the critical behavior near the phase transitions is presented in Sec. V] The interesting behavior of the energy gaps is discussed in Sec. VI and we conclude in Sec. VII

\section{MODEL}

The frustrated honeycomb model shown in Fig. 1 is described by the spin-1/2 Hamiltonian

$$
\mathcal{H}=\mathcal{H}_{\text {legs }}+\mathcal{H}_{\text {rungs }}
$$

with

$$
\mathcal{H}_{\text {legs }}=\sum_{\alpha=1,2} \sum_{j=1}^{L}\left(J_{1} \mathbf{S}_{j, \alpha} \cdot \mathbf{S}_{j+1, \alpha}+J_{2} \mathbf{S}_{j, \alpha} \cdot \mathbf{S}_{j+2, \alpha}\right)
$$

where $\alpha=1,2$ are the leg indices, and the interleg exchange on the odd rungs reads

$$
\mathcal{H}_{\text {rungs }}=J_{\perp} \sum_{j=1}^{L} \frac{1-(-1)^{j}}{2} \mathbf{S}_{j, 1} \cdot \mathbf{S}_{j, 2} \text {. }
$$

Frustrating coupling $\left(J_{\times}\right)$between the legs can in principle also be included but will not be considered here. The leg couplings $J_{1}, J_{2}>0$ are both chosen to be antiferromagnetic, while the rung couplings $J_{\perp}$ can be ferromagnetic or antiferromagnetic. For couplings on every rung the known instabilities are either the $\mathrm{RS}$ phase or the Haldane phase, depending on the sign, ${ }^{23-28}$ but the alternation may open the possibility for a richer phase diagram especially in connection with the frustration $J_{2}$, which tends to induce ordered dimerized phases $\underline{34-41}$

Obviously, the total spin $\mathbf{S}_{t}=\sum_{j, \alpha} \mathbf{S}_{j, \alpha}$ commutes with model Hamiltonian, due to the global $S U(2)$ symmetry. There is a mirror symmetry under exchange of the leg indices. For periodic boundary conditions (PBC) the translational symmetry by two rungs is maintained, but there is no parity symmetry along the legs. Only for an odd number of rungs $L$ and open boundary conditions (OBC) such a parity symmetry exists with respect to the middle rung.

\section{GROUND STATE PHASE DIAGRAM}

In this section, the quantum phase diagram is summarized and discussed before the underlying detailed analytical and numerical calculations are presented in the following sections. The phase diagram in Fig. 2

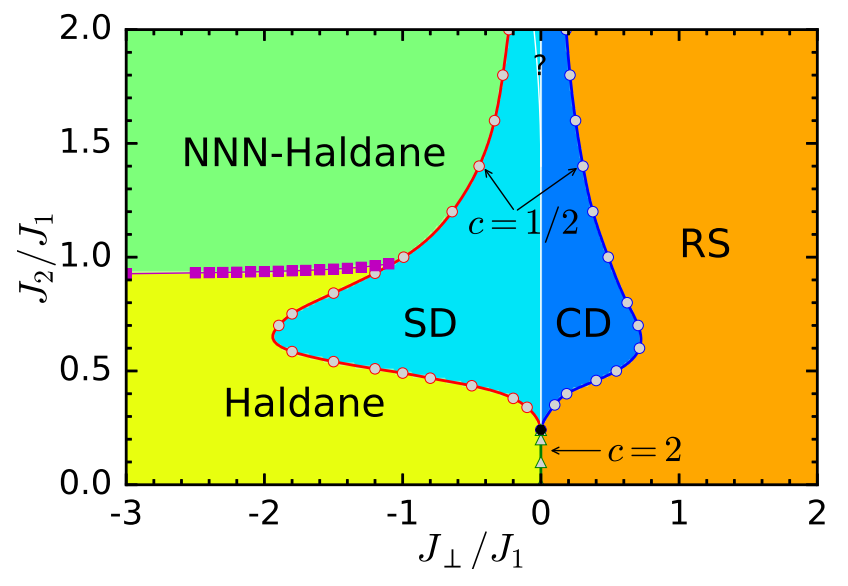

FIG. 2. Ground state phase diagram of the frustrated twoleg honeycomb ladder. The ordered dimer phases SD and CD are separated by a first order transition and Haldane to NNNHaldane is also of weak first order. All other phase transitions are continuous $(\circ: c=1 / 2 ; \Delta: c=2)$.

was derived by extensive density matrix renormalization group (DMRG) ${ }^{52-55}$ and infinite-size density matrix renormalization group (iDMRG) $)^{56-58}$ methods with the $U(1)$ symmetry. OBC and shifted OBC (see appendix B) are used unless stated explicitly otherwise. The numerical calculations are implemented by keeping states in both blocks up to 2000 for letting the truncation error less than $10^{-7}$. For the DMRG, larger than four iterative steps of sweep are used to guarantee the convergence of the ground state and the low-lying excited states. In the iDMRG simulation, a warm-up process with at least 1000 truncated states is used and the number of states $m$ increases during the measurement.

The model Hamiltonian in Eq. (1) allows to define two parameters, which adjust the frustration $J_{2} / J_{1}$ in the legs and the coupling $J_{\perp} / J_{1}$ between the legs independently. The resulting phase diagram in Fig. 2 shows five extended phases: Haldane, NNN-Haldane, and staggered dimer (SD) for $J_{\perp}<0, \mathrm{CD}$ and $\mathrm{RS}$ for $J_{\perp}>0$. There is also a TLL phase along the line of $J_{\perp}=0$ and $J_{2}<J_{2, c}$.

In the strong ferromagnetic limit of the odd-rung coupling $-J_{\perp} \gg J_{1}$, the spins on the odd rungs are in the triplet state, which effectively behave as spin-1. The NN interaction $J_{1}$ to the spins in the rung-alternating model may in principle cause interesting states with broken translational symmetry, where effective triplets over several rungs may be formed (so-called Haldane-dimer states) $4,4,59$ However, for the simple alternation of bonds in Eq. (3) the states on the even rungs are always observed to be in an effective triplet state in agreement with second order perturbation theory for $-J_{\perp} \gg J_{1}$. In particular, for relatively strong $\mathrm{NN}$ coupling $J_{1} \gg J_{2}$ the triplets on the even rungs can form valence bonds to the odd rungs on both sides equally, which corresponds to the well-known Haldane state. For the spin-1 chain this state was shown to be topologically nontrivial and protected by time-reversal, parity and dihedral group sym- 
metries (incomplete $\left.Z_{2} \times Z_{2}\right) \stackrel{60,61}{=}$ For OBC, topologically protected spin- $1 / 2$ degrees of freedom are localized at the edges. Thus it is well characterized by a double degeneracy in the entanglement spectrum. ${ }^{62}$ It is also interesting that the translational symmetry by one-rung is recovered since no dimerization is observed in such a Haldane phase. On the other hand, in the limit $J_{2} \gg J_{1}$ a NNN-Haldane state is formed only involving the odd rungs directly. The spins on the even rungs then again form induced triplet states, which are also stable in a NNN-Haldane state. In this case, the translation by one rung remains broken. This state is therefore characterized by two weakly coupled Haldane states with valence bonds over NNN. The fractional edge spin- $1 / 2$ are no longer protected, so the state can adiabatically transit to a trivial state without breaking any symmetry. Analogous to the $J_{1}-J_{2}$ spin- 1 chain, $\stackrel{15-22}{2}$ a weak first order topological phase transition from the Haldane phase to NNN-Haldane phase can be observed.

For weaker ferromagnetic rung coupling there is a more complex competition between frustration and triplet formation. Above a critical value which can be predicted from bosonization in the next section, the frustrating coupling $J_{2}$ causes a long-range dimer order in each of the legs. The weak ferromagnetic odd-rung coupling then causes a staggered pattern of dimers on the two legs 41 (SD phase). In contrast to the Haldane phase this state is long-range ordered with a broken translational symmetry by one rung. The region of the SD phase extends far to the ferromagnetic side, especially around $J_{2} \approx 0.6 J_{1}$ where the dimerization and triplet gap of the underlying zigzag chains are largest $\underline{\underline{7}}$ However, the transition from Haldane to NNN-Haldane occurs at even larger $J_{2}$, which in turn causes an interesting reentrant behavior around moderate values of $J_{\perp} \sim-1.5 J_{1}$ : Increasing $J_{2}$ causes a phase transition from Haldane to SD and then again from SD to Haldane states, before finally the NNN-Haldane phase is observed at larger $J_{2}$.

In the limit of strong antiferromagnetic inter-leg coupling $J_{\perp} \gg J_{1}$, singlets are formed on the odd rungs. Also on the even rungs an effective antiferromagnetic correlation is induced via second order perturbation theory, so that a so-called rung singlet state $\underline{\underline{23}}-\underline{28}$ with short range correlations is found. Slightly stronger couplings along the legs cause resonating valence bonds $\frac{25}{2}$ but do not change the nature of the correlations. Therefore, in contrast to strong ferromagnetic rung couplings there are no degrees of freedom available which could allow another phase in the limit $J_{\perp} \gg J_{1}$. For weak antiferromagnetic rungs, on the other hand, the dimerized state becomes important again. For uncoupled legs dimerization is stable for $J_{2}>J_{2, c^{3}} \underline{\underline{-1}}$ and the weak antiferromagnetic rungs now cause a columnar dimer pattern on the two legs: $\stackrel{41}{=}$ The region of the resulting $C D$ phase is again largest around $J_{2} \approx 0.6 J_{1}$ where the strongest dimer correlations and triplet gaps are found $\underline{\underline{7}}$ However, the CD phase only extends about a third in $\left|J_{\perp}\right|$ compared to the SD phase, which is simply due to the fact that breaking rung singlets costs three times the energy compared to the triplets. For small $J_{\perp}$ the shape of the transition line can again be predicted by bosonization in the next section, which is found to be in the 2D Ising universality class. Incommensurate short range correlations are found in the $\mathrm{SD}$ and $\mathrm{CD}$ phases for larger frustration $J_{2}$, but the dimerization order parameter changes continuously ${ }^{7}$ The transition line between SD and CD phases has been drawn at $J_{\perp}=0$ in Fig. 2, since obviously the SD and CD pattern must be degenerate for uncoupled chains. However, it must be emphasized that there is a large uncertainty if the $\mathrm{CD}$ phase may also be stable for small negative $J_{\perp}$ in a region around $J_{2} \sim 1.5 J_{1}$, which both bosonization and numerical results seem to indicate as discussed in Secs. IV and V.

\section{FIELD THEORY AND RENORMALIZATION GROUP}

A RG treatment based on bosonization gives an analytical approach to determine the phase boundaries $\frac{7.29,31,40,41}{4}$ Here we employ non-Abelian bosonization to express the spin operators in an effective continuum theory of decoupled chains ${ }^{3,64}-66,68$

$$
\mathbf{S}(x) \approx \mathbf{J}(x)+(-1)^{x} \Omega \mathbf{n}(x),
$$

with $\Omega$ being a nonuniversal constant of order of one. The uniform part of the spin operator $\mathbf{J}=\mathbf{J}_{L}+\mathbf{J}_{R}$ is the sum of the chiral $\mathrm{SU}(2)$ currents of the Wess-Zumino-Witten (WZW) model, while the staggered one is related to the matrix field $g$ of the WZW model via $\mathbf{n} \sim \operatorname{Tr} \boldsymbol{\sigma} g$, with $\boldsymbol{\sigma}$ the Pauli matrices. By symmetry, the field $\epsilon \sim \operatorname{Tr} g$ is also allowed corresponding to the dimerization operator, $\epsilon_{j}=$ $(-1)^{j} \mathbf{S}_{j} \cdot \mathbf{S}_{j+1}$. In what follows we consider this theory for fixed points of decoupled chains, which are perturbed by the interchain couplings. The RG treatment is then used to identify the leading instabilities, which in turn determine the phase transition lines.

Let us first consider the fixed point of $J_{\perp}=0$ and $J_{2}<J_{2, c}$, corresponding to two decoupled gapless chains (black lines in Fig. (1). It is well known that even without inter-leg coupling there is a marginal perturbation from an in-chain current-current marginal operator ${ }^{67}$ for each $\operatorname{leg} \alpha=1,2$

$$
\mathcal{H}_{a}=2 \pi v \int d x \lambda_{a} O_{a}, \quad O_{a}=\sum_{\alpha} \mathbf{J}_{\alpha, L} \cdot \mathbf{J}_{\alpha, R}
$$

of scaling dimension $d_{a}=2$. The velocity $v \approx \pi J_{1} / 2-$ $1.65 J_{2}$ as well as the bare coupling $\lambda_{a} \approx 1.723\left(J_{2}-J_{2, c}\right)$ can be found as functions of the in-chain NNN coupling $J_{2} 2^{3,6,68}$ The inter-leg couplings connect the chain field theories yielding a number of perturbations to the fixed point Hamiltonians, which in principle contain all operators allowed by symmetry. The possible perturbing operators with the lowest scaling dimensions are found 


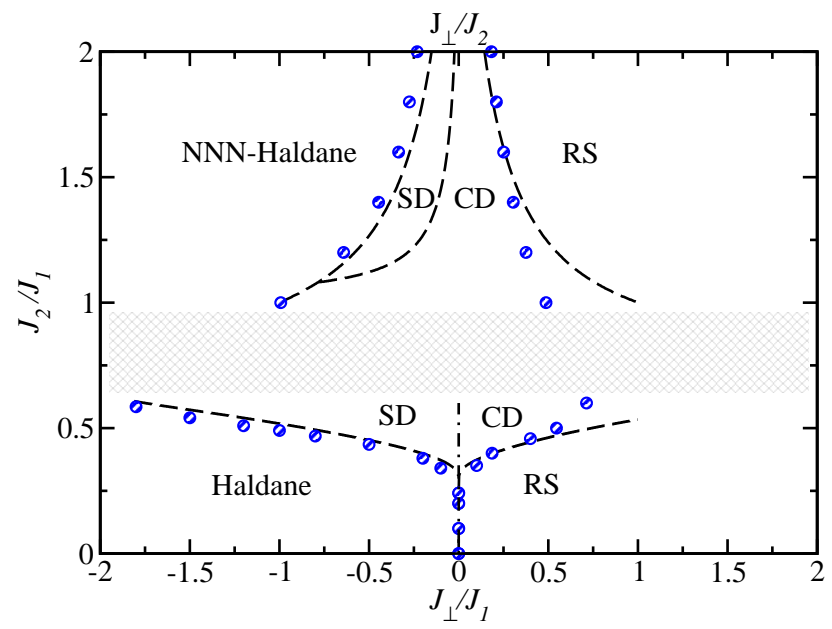

FIG. 3. Ground state phase diagram of the honeycomb ladder obtained via the field theory and RG. The blue circles correspond to the critical points determined numerically via the DMRG algorithm, while the black dashed lines indicate the critical lines as determined via analytical $\mathrm{RG}$ using $\Omega=\pi$ and $\lambda_{*}=1$.

to be

$$
\mathcal{H}_{1}=2 \pi v \int d x\left(\lambda_{\epsilon} O_{\epsilon}+\lambda_{n} O_{n}+\lambda_{c} O_{c}+\lambda_{b} O_{b}\right)
$$

with

$$
\begin{aligned}
& O_{\epsilon}=\epsilon_{1} \epsilon_{2}, O_{n}=\mathbf{n}_{1} \cdot \mathbf{n}_{2}, O_{c}=\mathbf{J}_{1} \cdot \mathbf{n}_{2}+\mathbf{J}_{2} \cdot \mathbf{n}_{1} \\
& O_{b}=\mathbf{J}_{1, L} \cdot \mathbf{J}_{2, R}+\mathbf{J}_{2, L} \cdot \mathbf{J}_{1, R}
\end{aligned}
$$

with scaling dimensions $d_{\epsilon}=d_{n}=1, d_{c}=3 / 2$, and $d_{b}=2$. The bare couplings are determined by using Eq. (4)

$$
\lambda_{\epsilon}=0, \quad \lambda_{n}=\Omega^{2} \frac{J_{\perp}}{4 \pi v}, \quad \lambda_{c}=\Omega \frac{J_{\perp}}{4 \pi v}, \quad \lambda_{b}=\frac{J_{\perp}}{4 \pi v} .
$$

Note that although $\lambda_{\epsilon}$ is initially zero, it can be generated under the RG evolution of the couplings due to the second order contribution of the relevant $O_{c}$ operator.

In the RG treatment the energy cutoff is lowered according to $\Lambda(l)=\Lambda_{0} e^{-l}$ with the dimensionless scale parameter $l$, which changes the effective coupling constants. ${ }^{66.69}$ The evolution of each coupling is described by $65,69,72$

$$
\frac{d \lambda_{k}}{d l}=\left(2-d_{k}\right) \lambda_{k}-\frac{\pi}{v} \sum_{i, j} C_{i j k} \lambda_{i} \lambda_{j}
$$

where the coefficients $C_{i j k}$ are determined by the operator product expansion between the perturbing operators and $d_{k}$ is the corresponding scaling dimension. In our case this results in the following RG equations (see ap- pendix $\mathrm{A}$

$$
\begin{aligned}
& \dot{\lambda_{a}}=\lambda_{a}^{2}+\frac{1}{2} \lambda_{\epsilon}^{2}-\frac{1}{2} \lambda_{n}^{2} \\
& \dot{\lambda_{b}}=\lambda_{b}^{2}-\lambda_{\epsilon} \lambda_{n}+\lambda_{n}^{2} \\
& \dot{\lambda_{\epsilon}}=\lambda_{\epsilon}+\frac{3}{2} \lambda_{a} \lambda_{\epsilon}-\frac{3}{2} \lambda_{b} \lambda_{n}+\frac{3}{2} \lambda_{c}^{2} \\
& \dot{\lambda_{n}}=\lambda_{n}-\frac{1}{2} \lambda_{a} \lambda_{n}-\frac{1}{2} \lambda_{b} \lambda_{\epsilon}+\lambda_{b} \lambda_{n}+\lambda_{c}^{2} \\
& \dot{\lambda_{c}}=\frac{1}{2} \lambda_{c}-\frac{1}{4} \lambda_{a} \lambda_{c}+\lambda_{b} \lambda_{c}+\frac{1}{2} \lambda_{c} \lambda_{\epsilon}-\lambda_{c} \lambda_{n}
\end{aligned}
$$

We now integrate these equations until the magnitude of one coupling constant reaches the cut-off $\lambda^{*}=1$, which in turn determines the dominant correlations. As the strong coupling regime is reached, the system is no longer scale-invariant and renormalization stops. When either $\lambda_{a}$ or $\lambda_{\epsilon}$ first reaches the strong coupling limit the ladder is dimerized, where $\lambda_{\epsilon}>0$ corresponds to an SD pattern while $\lambda_{\epsilon}<0$ gives the CD phase. When either $\lambda_{b}$ or $\lambda_{n}$ becomes dominant the system acquires strong correlations on the rungs, where $\lambda_{n}>0$ corresponds to singlets (RS) and $\lambda_{n}<0$ to triplets (Haldane). There is no region in the parameter space where the less relevant operator $O_{c}$ becomes dominant, and its role is restricted to simply promote one of the other phases. If this operator can be made large by fine-tuning in an extended parameter space, it was argued to enhance incommensurate correlations, 41 The lower part of the phase diagram in Fig. 3 was produced by identifying the coupling constant to first reach the values $\lambda_{*}=1$ under integration of the RG equations, where $\Omega=\pi$ was used for the best agreement with the DMRG results. Both $\lambda_{*}$ and $\Omega$ are in principle adjustable parameters, which change the exact location of the lines, but not the topology of the phases.

There is another possible fixed point in the limit $J_{2} \gg$ $J_{1}, J_{\perp}$, which consists of four decoupled chains denoted by $\bar{\alpha}=1,2,3,4$ corresponding to the red lines in Fig. 1 . In this case, the couplings between the inner and the outer chains are different depending on $J_{1}$ and $J_{\perp}$. Consequently, we need to treat the marginal operator from Eq. (5) separately for the the inner chains $(\bar{\alpha}=2,3), O_{i}$ with coupling $\lambda_{i}$; and for the outer ones $(\bar{\alpha}=1,4), O_{o}$ with coupling $\lambda_{o}$. The interchain perturbation now reads

$$
\begin{aligned}
\mathcal{H}_{2}=2 \pi v \int d x\left[\lambda_{\epsilon} O_{\epsilon}\right. & +\lambda_{n} O_{n}+\lambda_{b} O_{b}+\lambda_{u} O_{u} \\
& \left.+\lambda_{v} O_{v}+\lambda_{w} O_{w}\right]
\end{aligned}
$$

with,

$$
\begin{aligned}
O_{\epsilon} & =\epsilon_{2} \epsilon_{3}, O_{n}=\mathbf{n}_{2} \cdot \mathbf{n}_{3}, \\
O_{b} & =\mathbf{J}_{2, L} \cdot \mathbf{J}_{3, R}+\mathbf{J}_{3, L} \cdot \mathbf{J}_{2, R}, \\
O_{u} & =\mathbf{J}_{1, L} \cdot \mathbf{J}_{2, R}+\mathbf{J}_{3, L} \cdot \mathbf{J}_{4, R}+L \leftrightarrow R, \\
O_{v} & =\epsilon_{1} \partial_{x} \epsilon_{2}+\epsilon_{4} \partial_{x} \epsilon_{3}, O_{w}=\mathbf{n}_{1} \cdot \partial_{x} \mathbf{n}_{2}+\mathbf{n}_{4} \cdot \partial_{x} \mathbf{n}_{3},
\end{aligned}
$$

with scaling dimensions $d_{\epsilon}=d_{n}=1$ and $d_{b}=d_{u}=d_{v}=$ 
$d_{w}=2$. The bare couplings

$$
\begin{aligned}
& \lambda_{\epsilon}=0, \lambda_{b}=\frac{J_{\perp}}{2 \pi v}, \lambda_{n}=\Omega^{2} \frac{J_{\perp}}{2 \pi v}, \\
& \lambda_{v}=0, \lambda_{u}=\frac{2 J_{1}}{2 \pi v}, \lambda_{w}=-\Omega^{2} \frac{J_{1}}{2 \pi v} .
\end{aligned}
$$

where the velocity is $v \approx \pi J_{2} / 2$. The resulting RG equations are

$$
\begin{aligned}
& \dot{\lambda}_{o}=\lambda_{o}^{2}-\frac{1}{4}\left(\lambda_{v}^{2}-\lambda_{w}^{2}\right), \\
& \dot{\lambda}_{i}=\lambda_{i}^{2}+\frac{1}{2}\left(\lambda_{\epsilon}^{2}-\lambda_{n}^{2}\right)-\frac{1}{4}\left(\lambda_{v}^{2}-\lambda_{w}^{2}\right), \\
& \dot{\lambda}_{b}=\lambda_{b}^{2}-\lambda_{n} \lambda_{\epsilon}+\lambda_{n}^{2}, \\
& \dot{\lambda}_{\epsilon}=\lambda_{\epsilon}+\frac{3}{2} \lambda_{i} \lambda_{\epsilon}-\frac{3}{2} \lambda_{b} \lambda_{n}, \\
& \dot{\lambda}_{n}=\lambda_{n}-\frac{1}{2} \lambda_{i} \lambda_{n}-\frac{1}{2} \lambda_{b} \lambda_{\epsilon}+\lambda_{b} \lambda_{n}, \\
& \dot{\lambda}_{u}=\lambda_{u}^{2}-\frac{1}{2} \lambda_{v} \lambda_{w}+\frac{1}{2} \lambda_{w}^{2}, \\
& \dot{\lambda}_{v}=\frac{3}{4}\left(\lambda_{o}+\lambda_{i}\right) \lambda_{v}-\frac{3}{2} \lambda_{u} \lambda_{w}, \\
& \dot{\lambda}_{w}=-\frac{1}{4}\left(\lambda_{o}+\lambda_{i}\right) \lambda_{w}-\frac{1}{2} \lambda_{u} \lambda_{v}+\lambda_{u} \lambda_{w} .
\end{aligned}
$$

For weak perturbations we find that the critical lines are determined by the competition of the relevant $O_{n}$, driving the system into strong rung correlations, and the marginal $O_{u}, O_{w}$ both of which drive the system into a dimerized phase for large values. The dimer operator $O_{\epsilon}$ is more relevant than the marginal couplings but remains smaller under RG flow for the starting values in Eq. (13). However, the pattern (SD or CD) is still determined by the sign of $\lambda_{\epsilon}$ which indicates that the transition line is shifted to negative values of $J_{\perp}$ in Fig. 3. It should be noted that the numerical analysis of the exact location of the transition line is also extremely difficult which will be discussed below in Sec. V, so it remains an open question if the CD phase may be stable for small negative $J_{\perp}$. All other transition lines are in quantitatively good agreement in the vicinity of both fixed points in Fig. 3. For larger bare couplings the RG approach breaks down, so no meaningful prediction can be made with bosonization in the shaded region of Fig. 3, which separates the analysis at the two fixed points.

\section{NATURE OF THE TRANSITIONS}

This section is devoted to discuss detailed numerical results for the nature of phase transitions presented in Sec. III. Traditional phase transition can be fully described in the framework of Landau theory of symmetry breaking, where the concept of local order parameter is involved. The critical exponents for the continuous phase transition can be extracted from the order parameter. However, a so-called symmetry protected topological (SPT) state, may only be characterized by non-local order parameter dubbed as topological order $\underline{\underline{73}}$

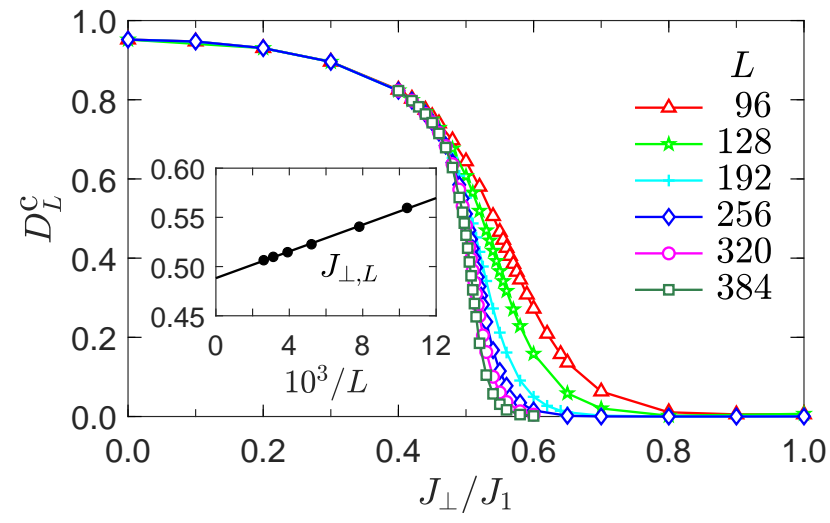

FIG. 4. Columnar dimerization order parameter $D_{L}^{\mathrm{c}}$ for OBC as a function of $J_{\perp}$ for $L=96(\Delta), 128(\aleph), 192(+), 256(\diamond)$, $320(\bigcirc)$ and $384(\square)$ when $J_{2}=J_{1}$ is fixed. In the inset, we do a linear extrapolation of the quasi-critical points $J_{\perp, L}(\bullet)$ to the TDL.

Since all the other disordered phases are adjacent to the dimerized phases in the parameter space considered, one can use the local order parameter of the $\mathrm{CD} / \mathrm{SD}$ phase to distinguish the phase boundaries and extract the critical exponents. The dimerization order parameters in the columnar and staggered patterns $D_{L}^{\mathrm{c}}$ and $D_{L}^{\mathrm{s}}$, respectively are given by

$$
D_{L}^{\mathrm{c} / \mathrm{s}}(j)=\sum_{\alpha=1,2}\left|\frac{( \pm 1)^{\alpha}}{2}\left[\left\langle\mathrm{~S}_{j, \alpha} \cdot \mathrm{S}_{j+1, \alpha}\right\rangle-\left\langle\mathrm{S}_{j-1, \alpha} \cdot \mathrm{S}_{j, \alpha}\right\rangle\right]\right| .
$$

The dimerization shows strong Friedel oscillations near edges, which decay exponentially towards the middle. Therefore we shall set $j=L / 2$ in Eq. (15) so as to minimize the boundary effect and chose compatible OBC as discussed in appendix B.

\section{A. RS-CD transition}

The typical behavior of the order parameter $D_{L}^{\mathrm{c}}$ with

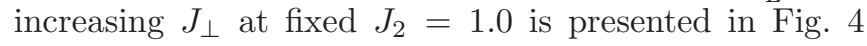
for different lengths $L$ and OBC. To determine the accurate value of the critical point $J_{\perp, c}$, we shall first obtain quasi-critical points $J_{\perp, L}$ which are defined by the position of the first-order derivative peaks of $D_{L}^{\mathrm{c}}$ for each length $L$. It is then possible to make an extrapolation to the thermodynamic limit (TDL) to find $J_{\perp, c}$ as shown in the inset of Fig. 4, which gives $J_{\perp, c} \simeq 0.4883(12)$ in this case. The signature of the phase transition can also be seen by local entanglement measures $\underline{74}$ and by the rungsinglet correlation in the RS phase $\underline{\underline{43}}$

Fundamental characteristics of ground states in quantum many-body systems are manifest in their entanglement properties. In particular, the von Neumann entropy has drawn much attention in condensed matter systems, which exhibits a universal scaling behavior and captures some criticality features $\underline{\underline{75}} \underline{\underline{79}}$ For example, the 


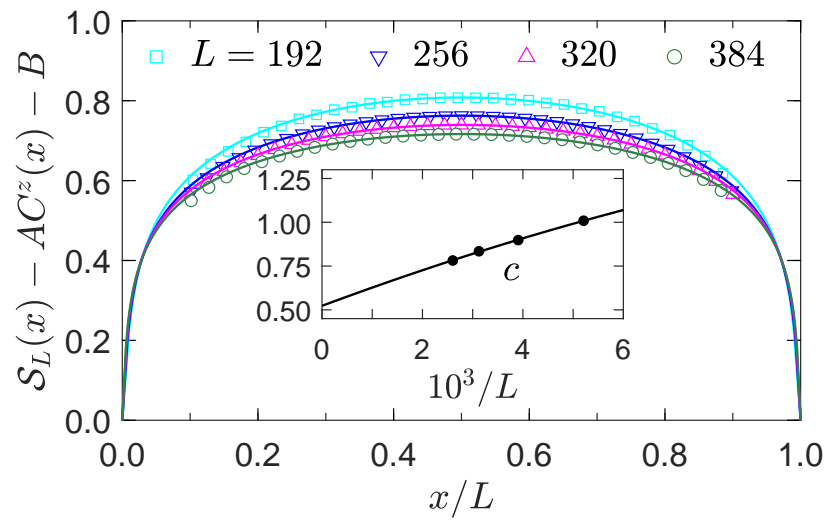

FIG. 5. Von Neumann entropy $\mathcal{S}$ as a function of partition size $x / L$ fitted by Eq. (16) at CD-RS quasi-critical points $J_{\perp, L}$ for different $L$. The inset shows a second-order polynomial extrapolation of the central charge to $c=0.52(3)$ in the TDL.

central charge describes the universality class of the criticality and sub-leading corrections, for critical points in

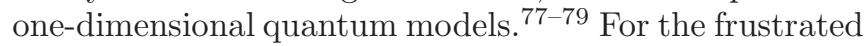
honeycomb ladder we use the scaling of the von Neumann entropy $\mathcal{S}_{L}(x)$

$$
\mathcal{S}_{L}(x)=\frac{c}{6} \ln \left[\frac{2 L}{\pi} \sin \left(\frac{\pi x}{L}\right)\right]+A C^{z}(x)+B,
$$

where $c$ is the central charge, $A$ and $B$ are non-universal fitting parameters, and $x$ is the size of the partition from the edge in units of the lattice spacing. Here $C^{z}(x)=C_{\alpha}^{z}(x) \equiv\left\langle S_{x, \alpha}^{z} S_{x+1, \alpha}^{z}\right\rangle$ is the $z$-component of the spin-spin correlation function along the leg direction, which is independent of the leg index $\alpha=1,2$ and alternates due to $\mathrm{OBC}$. Its relation to the alternating term of the von Neumann entropy under OBC was firstly noted by Wang, 80 and then demonstrated numerically by Laflorencie et al $\underline{81}$ Recently, the fluctuations of the spin-spin correlation function for the spin- $1 / 2 X X$ chain were calculated analytically, which show that the sub-leading terms of the oscillations exist $\underline{82,83}$

In order to obtain a rather accurate central charge $c$ defined in Eq. (16), some tricks can be employed to reduce the finite size effects and the rapid oscillations due to the $\mathrm{OBC}$ as well as the alternating nature of the honeycomb ladder. We calculate the central charge at the quasicritical points $J_{\perp, L}$ for each length $L$ and extrapolate, as shown in Fig. 5. We stress here that each quasi-critical point $J_{\perp, L}$ happens to be the position of the maximum value of von Neumann entropy. The resulting central charge $c \simeq 0.52(3)$ indicates that the phase transition RS-CD has the universality class of the 2D Ising type with an exact central charge of $c=1 / 2 \underline{66}$

We now take a closer look at the energy gaps near the phase transitions. The ground state can always be found in the sector with total spin- $z S_{t}^{z}=0$. The triplet gap $\Delta_{T}$ is determined numerically by the energy difference to the first excited state with $S_{t}^{z}=1$. We can also find

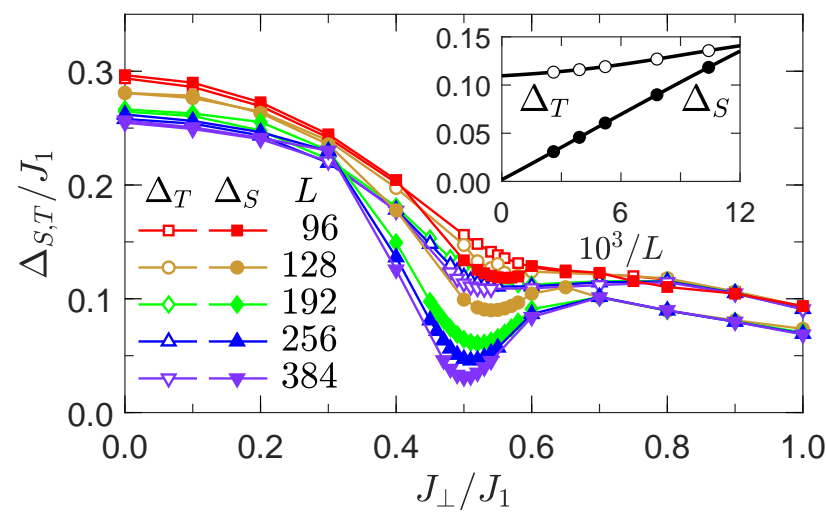

FIG. 6. Evolution of singlet gap $\Delta_{S}$ (filled symbols) and triplet gap $\Delta_{T}$ (open symbols) as a function of $J_{\perp}$ when $J_{2}=$ 1.0 for $L=96,128,192,256$ and 384. The inset gives a linear fit of $\Delta_{S}(\bullet)$ and a second-order polynomial fit $\Delta_{T}($ ( ) at the quasi-critical points to the TDL.
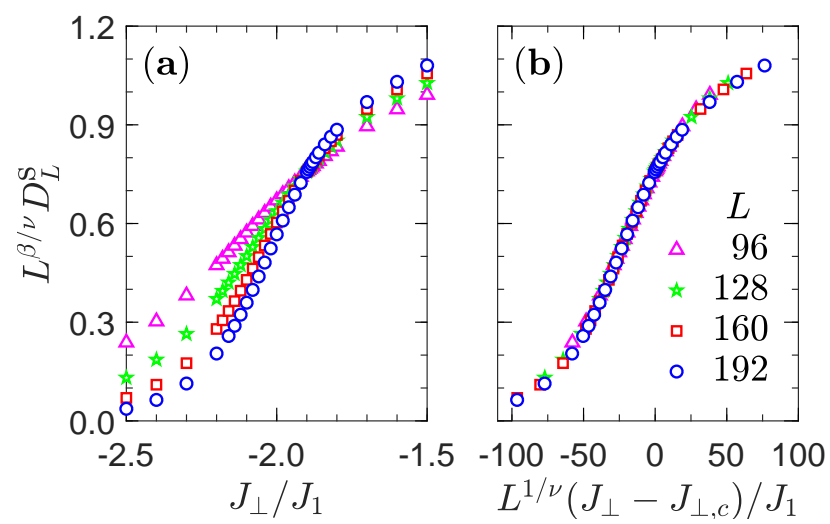

FIG. 7. FSS analysis of the staggered dimerization order parameter $D_{L}^{\mathrm{s}}$ for $J_{2}=0.7 J_{1}$ as a function of $J_{\perp}$ for different length $L=96(\Delta), 128(\hat{\xi}), 160(\square)$ and $192(\bigcirc)$. The best fitting suggests $J_{\perp, c}=-1.8980(5)$ and critical exponents $\beta=0.126(2)$ and $\nu=0.98(2)$.

the singlet gap $\Delta_{S}$ to the first excited state with $S_{t}^{z}=0$. We show the value of gaps for various sizes $L$ in Fig. 6 . where for each $L$ the local minimum of the singlet gap corresponds to the quasi-critical point $J_{\perp, L}$ in Fig. 5. An extrapolation of the gaps at quasi-critical points to the TDL is shown in the inset. When $J_{\perp}$ crosses the critical point, the triplet gap remains finite while the singlet gap tends to be zero.

\section{B. Haldane/NNN-Haldane-SD transition}

The transition between the Haldane/NNN-Haldane phase and the SD phase is similar to that of the RS$\mathrm{CD}$ transition. The phase transition could be found as before, but instead we want to take the opportunity to illustrate that a finite-size-scaling (FSS) method with critical indices gives a good data collapse. In particular, the 


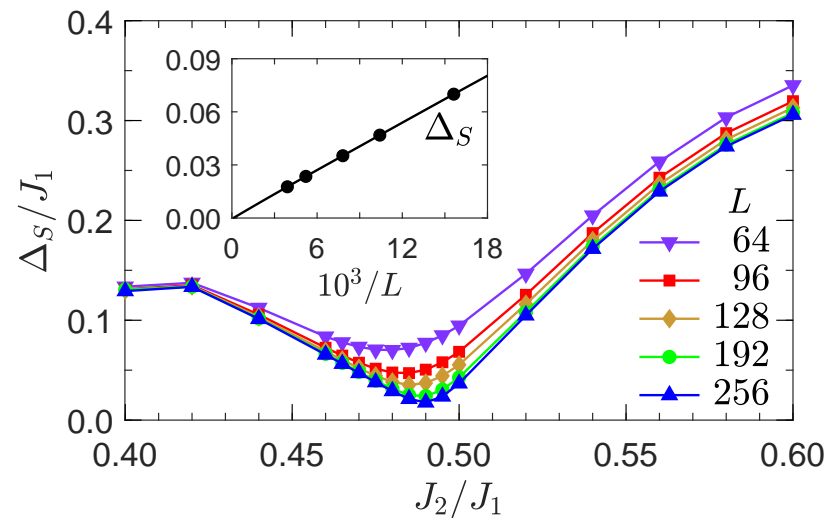

FIG. 8. Evolution of the singlet gap for shifted OBC in the vicinity of the critical point $J_{2, c} \simeq 0.5$ at fixed $J_{\perp}=-1.0$ for $L=64(\nabla), 96(\square), 128(\diamond), 192(\bigcirc)$ and $256(\Delta)$. In the inset, a linear extrapolation of the singlet gap $\Delta_{S}$ at quasicritical points goes to zero in the TDL.

behavior of the order parameter $D_{L}^{s}$ with the finite size $L$ follows

$$
D_{L}^{s}(J) \simeq L^{-\beta / \nu} f_{D}\left(\left|J-J_{c}\right| L^{1 / \nu}\right),
$$

where the critical exponent $\nu$ of the gap describes the divergence of the correlation length while $\beta$ is the critical exponent of the order parameter $D \sim\left|J-J_{c}\right|^{\beta}$. To obtain critical exponents, we change exponents $\mu_{1,2}$ until we see the collapse of $D_{L}^{s}(J) L^{\mu_{1}}$ as a function of $\left|J_{\perp}-J_{\perp, c}\right|^{\mu_{2}}$ for all lengths $L$. In this manner we extract $\beta=\mu_{1} / \mu_{2}$ and $\nu=1 / \mu_{2}$.

As an example, the FSS of the order parameter $D_{L}^{\mathrm{s}}$ for $J_{2}=0.7$ is shown in Fig. 7 for the SD-Haldane transition. From the scaling of $D_{L}^{\mathrm{s}}$ we obtain the critical point $J_{\perp, c} / J_{1} \simeq-1.8980(5)$, critical exponents $\beta=0.126(2)$ and $\nu=0.98(2)$. These values coincide well with the critical indices of the $2 \mathrm{D}$ Ising universality class where $\beta=1 / 8$ and $\nu=1$. Following the same way introduced in the previous subsection, we get the central charge at the critical point $J_{\perp, c}, c=0.51(2)$, which is another independent evidence of the 2D Ising universality class.

We now turn to the topological properties of the Haldane and SD phases by analyzing the entanglement spectrum $\xi_{\chi}$, which is derived from DMRG eigenvalues $w_{\chi}$ of the reduced density-matrix $\rho_{A}$ for the right half of the system if the degrees of freedom in the other half are traced out, namely $\xi_{\chi}=-\ln \left(w_{\chi}\right) \underline{\underline{84}}$ It has been shown that the entanglement spectrum of a SPT state exhibits a nontrivial multiplet structure of even-fold degeneracy ${ }^{62}$ However, the multiplet structure is influenced by the ladder structure and is strongly dependent on the boundary condition. For example, the one-dimensional spin-1 Heisenberg chain is gapped with a unique ground state and a four-fold degeneracy of the entanglement spectrum under PBC. For OBC the edge states give rise to a fourfold degenerate ground state in the TDL and a two-fold degeneracy of the entanglement spectrum $\stackrel{62}{ }$ For the hon-

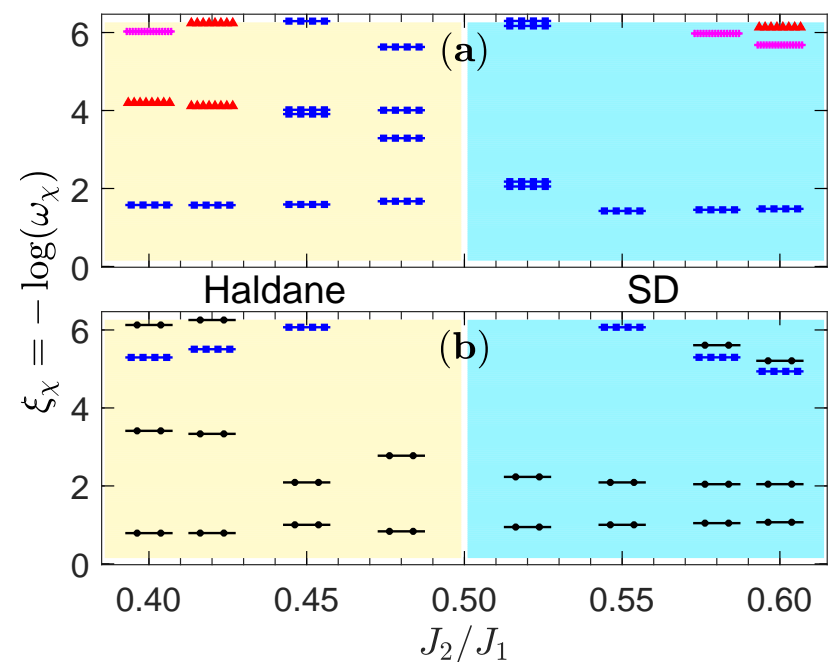

FIG. 9. Lower part of the entanglement spectrum of the Haldane and SD phases in the vicinity of the critical point $J_{2, c} \simeq 0.5$ at fixed $J_{\perp}=-1.0$ for (a) $L=64$ with PBC (b) $L=95$ with shifted OBC (see appendix B). In the multiplet structure the degeneracy is indicated by different symbols: 2 (black circles), 4 (blue squares), 8 (red triangles) and 16 (magenta plus).

eycomb ladder the choice of OBC is therefore quite important as also discussed in appendix $\mathrm{B}$. In both SD and Haldane phases with OBC, the ground state would generate two spin- $1 / 2$ confined at the edges. By a relative shift of the legs by one site (shifted OBC) it is possible to provide additional edge spins as discussed in appendix B In this case the ground state is unique and the entanglement spectrum has no spurious degeneracy. This can be verified by the nonzero spin singlet gap $\Delta_{S}$ for an interchain coupling $J_{\perp}=-1.0$ as a function of NNN coupling $J_{2}$ in Fig. 8. Near the critical point an avoided level crossing phenomenon emerges, where the gap shows a local minimum with linear finite size behavior towards zero as shown in the inset. The entanglement spectrum recovers an even-fold degeneracy as shown Fig. 9(b). In Fig. 9(a) the entanglement spectrum for PBC is shown. Remarkably, the degeneracy of the entanglement spectrum in the SD phase is also even-fold and therefore we cannot distinguish the two phases solely through the entanglement spectrum.

\section{Haldane-RS transition}

Haldane and RS phases are both disordered and uniform without breaking any time-reversal, parity and point group symmetries. Here the Haldane phase is a topologically nontrivial state with finite string order and edge spins, while the RS state does not have edge spins, which is due to the different symmetry of the corresponding string order parameter $\underline{\underline{31}}$ The direct transition between the Haldane phase and RS phase is continuous 


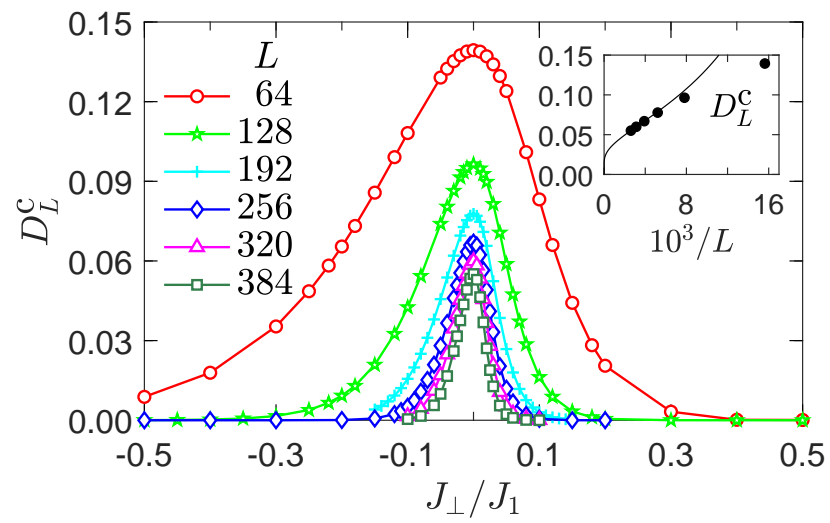

FIG. 10. Columnar dimerization order parameter $D_{L}^{\mathrm{c}}$ as a function of $J_{\perp}$ at fixed $J_{2}=0.2$ for $L=64(\bigcirc), 128(\hbar)$, $192(+), 256(\diamond), 320(\Delta)$ and $384(\square)$. Inset shows the extrapolation of maximal values using $D_{L}^{\mathrm{c}} \rightarrow 0.1268 / \ln (L / 38.5)$ as $L \rightarrow \infty$.

with central charge $c=2$, which has been the topic of many works $\stackrel{23-29}{-29}$ To show that there is not an intermediate dimerized phase for $J_{2}<J_{2, c}$, we illustrate the behavior of the order parameter of the dimerized phase across the transition in Fig. 10 for $J_{2}=0.2 J_{1}$. Without loss of generality, hereafter we shall only consider OBC and let $J_{2}=0.2 J_{1}$ throughout the subsection. For each given length $L$, the maximal value $D_{L}^{\mathrm{c}}$ is always exactly at $J_{\perp}=0$. The extrapolation of quasi-critical points $J_{\perp, L}$ on both sides show that the critical point is $J_{\perp}=0$ within the limit of error. The maximal values $D_{L}^{\mathrm{c}}$ in the inset approach the TDL on a non-linear curve, which is consistent with a logarithmically slow $D_{L}^{c} \sim 1 / \ln \left(L / L_{0}\right)$ decrease as $L \rightarrow \infty$ in agreement with the RG flow of the corresponding marginal operator in Eq. (5) $)^{\underline{3}-7,68,85}$

The phase transition is also reflected in the von Neumann entropy $\mathcal{S}_{L}$, which is shown in Fig. 11] for different lengths $L$ of the system. For each given $L, \mathcal{S}_{L}$ has a peak for the antiferromagnetic $\left(J_{\perp}>0\right)$ and ferromagnetic $\left(J_{\perp}<0\right)$ coupling, respectively. At the decoupled case $\left(J_{\perp}=0\right)$, a local minimum emerges. With increasing length $L$, positions of the peaks $J_{\perp, L}^{ \pm}$on both sides converge to the symmetric point $J_{\perp, c}=0$ where the continuous phase transition occurs (see inset). We also obtain the central charge $c$ from the von Neumann entropy $\mathcal{S}_{L}$. Best fitting with Eq. (16) for different sizes $L$ at the point $J_{\perp}=0$ suggests $c=1.98(3)$, which is in agreement with the existence of two decoupled TLL with $c=1$ each for $J_{\perp}=0$.

\section{Haldane-NNN-Haldane transition}

The spin-1 chain with NNN interactions was studied in previous works,$\stackrel{15-22}{\underline{2}}$ where a weak first order transition between the topological Haldane phase and the NNNHaldane phase was found. The situation in the honeycomb ladder with the strong-ferromagnetic odd rungs is

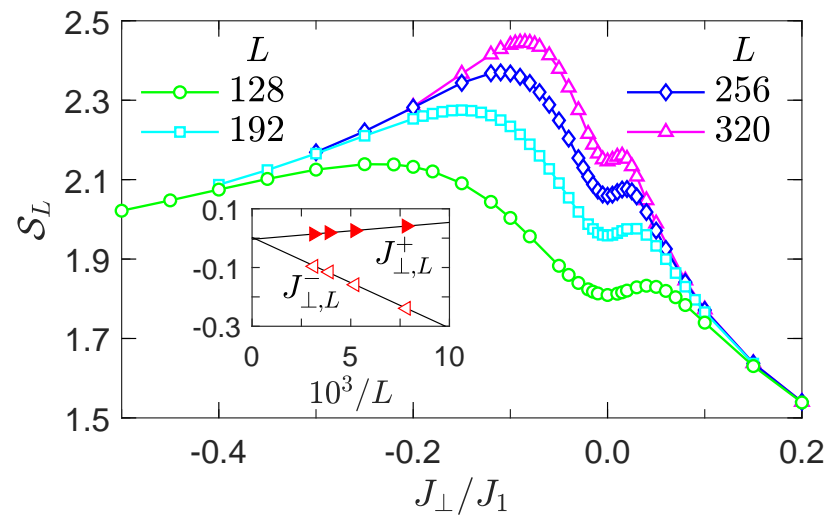

FIG. 11. Von Neumann entropy $\mathcal{S}_{L}$ as a function of $J_{\perp}$ at fixed $J_{2}=0.2$ for $L=128(\bigcirc), 192(\square), 256(\diamond)$ and $320(\Delta)$. In the inset, linear extrapolations of peak-positions $J_{\perp, L}^{ \pm}$on both sides approach the same critical point $J_{\perp, c}=0$ in the TDL.

slightly different, however, since triplet correlations on the even rungs are only indirectly induced by second order perturbation theory. Therefore, a careful analysis of this phase transition must be made to exclude the possibility of intermediate phases $\underline{\underline{44,59}}$

We now use the iDMRG method, $\underline{56-58}$ where no finitesize effect is involved. Instead of finite size scaling, an extrapolation in the number of kept state $m$ is useful so we vary this control parameter from 512 to 4096. The behavior of the von Neumann entropy $\mathcal{S}$ with frustration $J_{2}$ for different kept states $m$ is shown in Fig. 12 , The entropy has a discontinuous behavior in the narrow regime $J_{2} \in(0.926,0.928)$ in all cases, which clearly signals a phase transition. To further pinpoint the transition point, we analyze the derivative of the energy from the left and from the right side in this region as shown in the inset, which shows a clear jump at the transition point $J_{2, c} \simeq 0.92720754$. We therefore find no sign of intermediate phases or continuous behavior, so that the transition is of first order.

\section{E. SD-CD phase transition}

SD and CD phases are both dimerized states with conventional order parameters in the framework of Landau theory of phase transitions. Since they break different point group symmetries, the transition between them should be first-order. However it is a surprisingly big challenge to determine the transition line just from the numerical calculations. The main reason for this is that the observed dimerization pattern in finite chains also strongly depends on the edge geometries of the OBC or shifted OBC (see appendix B). To minimize the edge effect we therefore use the iDMRG method again. During the warm-up process both $\mathrm{OBC}$ and shifted OBC are used, until the average ground-state energy per site $e_{0}$ reaches very good convergence. Very close to $J_{\perp}=0$ the 


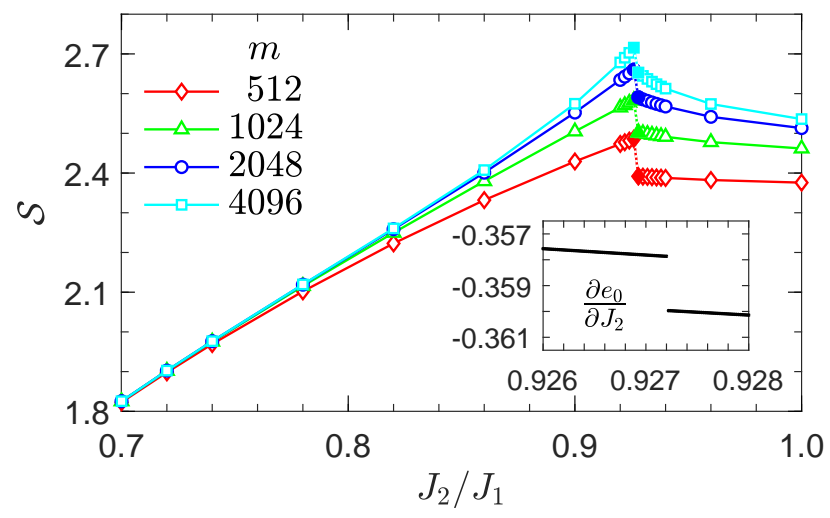

FIG. 12. Von Neumann entropy as a function of $J_{2}$ when fixed $J_{\perp}=-3.0$ for the Haldane-NNN-Haldane phase transition. We use $m=512(\diamond), 1024(\Delta), 2048(\bigcirc)$ and $4096(\square)$ in the iDMRG calculations. The inset shows the first-derivative of the average energy $e_{0}$ per site with respect to $J_{2}$ when $m=4096$.

shifted $\mathrm{OBC}$ always result in an SD phase, while $\mathrm{OBC}$ give the $\mathrm{CD}$ phase, albeit with slightly different energies $e_{0}^{s}$ and $e_{0}^{c}$, respectively. To identify the true ground state at zero temperature, we then analyze the energy difference $\Delta e_{0}=e_{0}^{c}-e_{0}^{s}$ per site. In Fig. [13, we show the results for three typical cases of $J_{2}=0.5,1.0$ and 1.5 and different states kept $m$. When $J_{2}=0.5$ and 1.0, the convergent $\Delta e_{0}$ always gives a positive sign on the left side $\left(J_{\perp}<0\right)$ and a negative sign on the right side $\left(J_{\perp}>0\right)$, so that the SD-CD transition appears to occur exactly at $J_{\perp}=0$. At $J_{2}=1.5$, the energy difference becomes very small and more than 10,000 states are required for convergence. The energy difference remains negative even for $J_{\perp} / J_{1}=-0.1$, but a clear position for the phase transition cannot be determined. It is also known that the dimerization becomes exponentially small for large $J_{2} \stackrel{7}{\underline{7}}^{\text {At }}$ this point it is unclear, if the phase transition remains at $J_{\perp}=0$ or if there is a region where the CD phase is stable at negative values of $J_{\perp}$ as also suggested by the bosonization analysis in Sec. IV] which will be an interesting but challenging topic for future research.

\section{ENERGY GAPS}

\section{A. A Special Case: $J_{2}=0$}

The honeycomb ladder is unique in that the couplings on even rungs are absent. Therefore, the correlations on the even rungs are only indirectly induced in second order corresponding to a weak energy gain. This has interesting consequences for the energy gaps. For example, in the ordinary ladder it is known that the triplet gap $\Delta_{T}$ increases linearly with larger rung coupling, $\underline{86,87}$ while we find that it decreases for stronger $J_{\perp}$ in the honeycomb ladder as shown in Fig. 14(d) for $J_{2}=0$. This behavior can in fact be understood by considering the subsystem

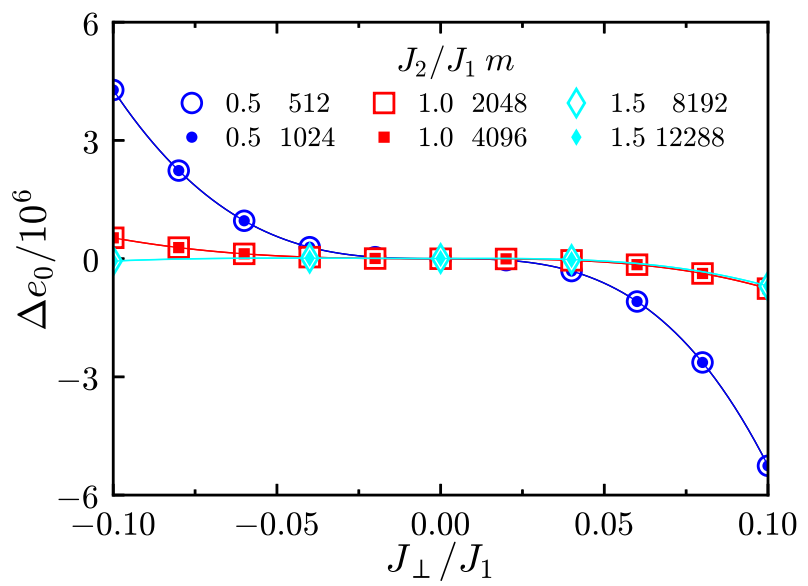

FIG. 13. Energy difference $\Delta e_{0}$ per site between $\mathrm{OBC}$ and shifted OBC given by iDMRG calculations. We choose three typical cases of $J_{2}=0.5(\bigcirc), 1.0(\square)$ and $1.5(\diamond)$ respectively. For each case, different truncated dimension in iDMRG is used from $m=512$ up to 12,288 . The error bar is smaller than the symbol size.

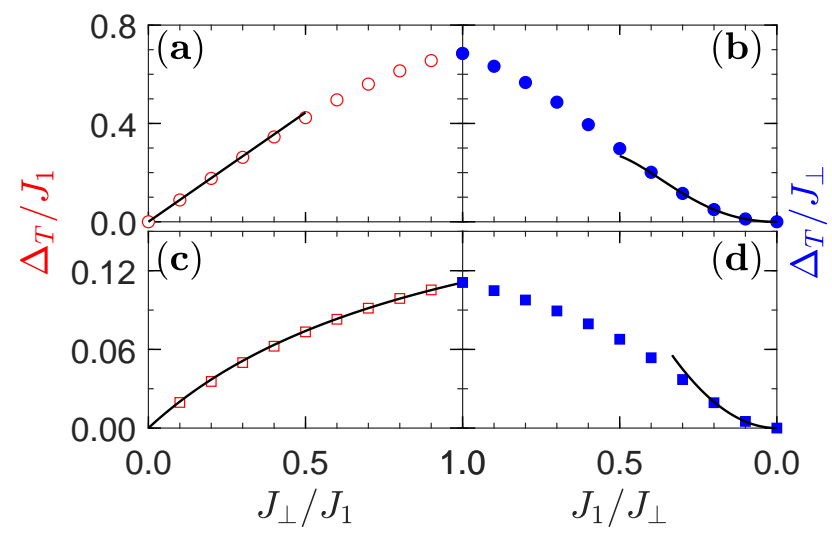

FIG. 14. Triplet gap $\Delta_{T}$ of the honeycomb cell $(\mathrm{a}, \mathrm{b})$ and the honeycomb ladder (c,d) for $J_{2}=0$ compared to strong coupling fits (solid lines).

of three adjacent rungs, which simply forms a ring of 6 spins coupled by $J_{1}$ and $J_{\perp}$ (honeycomb cell). The behavior of the triplet gap is analogous to the honeycomb ladder as shown in Fig. 14

For $J_{1} \gg J_{\perp}$ doublet states are formed by the top three and bottom three spins in the honeycomb cell, which in turn are coupled by $J_{\perp}$, so the triplet gap increases linearly with $\Delta_{T} \simeq 8 J_{\perp} / 9$ in Fig. 14(a). For the honeycomb ladder we also find a linear behavior, which fits well to the expression

$$
\frac{\Delta_{T}}{J_{1}} \approx 2 \Delta_{0} \frac{J_{\perp}}{J_{\perp}+J_{1}}
$$

with $\Delta_{0}=0.1110382(1)$.

In the opposite limit of $J_{1} \ll J_{\perp}$ the singlet-triplet gap in the honeycomb cell is produced by an indirect coupling 


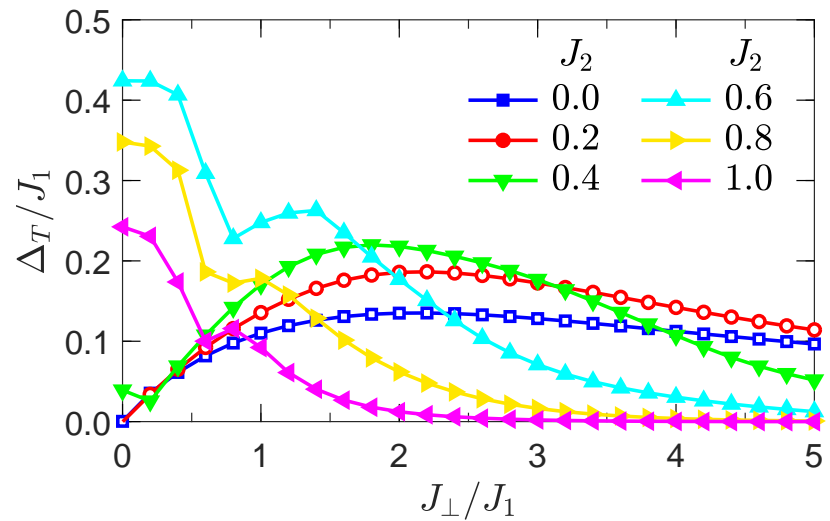

FIG. 15. Evolution of the triplet gap $\Delta_{T}$ for different $J_{2}$ in the TDL.

of the two spins of the central (uncoupled) rung, which can be found by a perturbation expansion

$$
\frac{\Delta_{T}}{J_{\perp}}=\lambda^{2}\left[1+\frac{3}{2} \lambda-\frac{3}{8} \lambda^{2}-\frac{75}{16} \lambda^{3}+\mathcal{O}\left(\lambda^{4}\right)\right]
$$

where $\lambda=J_{1} / J_{\perp}$. This also explains the drop of the triplet gap in the honeycomb ladder for large $J_{\perp}$, which follows $\Delta_{T} \simeq \frac{1}{2} J_{1}^{2} / J_{\perp}$ in that limit.

\section{B. $J_{2}>0$}

We now turn on the frustrating coupling $J_{2}$ to see if the non-monotonic behavior of the triplet gap with $J_{\perp}$ changes. The numerical results of different $J_{2}$ are shown in Fig. 15] For small $J_{2}<J_{2, c}$, the quick increase and slow drop-off with $J_{\perp}$ gives a broad maximum analogous to the $J_{2}=0$ case above. On the other hand, for larger $J_{2}>J_{2, c}$ the triplet gap is already nonzero for $J_{\perp}=$ 0 and then first drops to a minimum before rising and falling again. The size and location of the minimum is directly related to value of the gap at $J_{\perp}=0$ which is known ${ }^{7}$ to have a maximum around $J_{2}=0.6 J_{1}$. In fact, the positions of the gap minima roughly coincide with the CD-RS transition line, which implies that $J_{\perp}$ reduces the gap in the CD phase, while the broad maximum is a signature of the RS phase. Moreover, in the RS phase at a given $J_{\perp}$ a maximum can always be observed as a function of $J_{2}$.

\section{CONCLUSIONS}

In summary, we systematically analyzed the spin- $1 / 2$ honeycomb ladder as a function of inter-leg coupling $J_{\perp}$ on the odd rungs and NNN frustration on the legs $J_{2}$. Detailed numerical results for the order parameters, the entanglement spectrum, and the energy gaps from DMRG and iDMRG give a complete picture of the phase transitions, which agree quantitatively with our analytical bosonization calculations.
For very strong ferromagnetic odd-rung couplings we find a weak first order transition as a function of $J_{2}$ between Haldane and NNN-Haldane phases analogous to the frustrated spin-1 chain $\underline{\underline{15}-22}$ However, the SD phase with staggered dimer order is stable for relatively large ferromagnetic odd rungs which pushes far into the Haldane phase and leads to a reentrant behavior as a function of $J_{2}$. For antiferromagnetic odd-rung coupling we find a transition from the CD phase with columnar dimer order to a RS phase with rung singlets as a function of $J_{\perp}$ and again a reentrant behavior as a function of $J_{2}$ which can be linked to the corresponding maximum of the triplet gap in the underlying zigzag chain around $J_{2} \approx 0.6 J_{1} \underline{\underline{7}}$ The transitions from the ordered dimer phases are always of the 2D Ising universality class.

The line $J_{\perp}=0$ is critical below $J_{2}<J_{2, c}$ and corresponds to a $c=2$ transition between Haldane and RS phases. For larger $J_{2}$ the $\mathrm{CD}$ and SD phases appear, which must be degenerate for $J_{\perp}=0$. However, numerical and bosonization results surprisingly find that the CD phase may be stable also for small negative values of $J_{\perp}$ around $J_{2} \approx 1.5 J_{1}$, which would imply a highly non-trivial behavior that calls for further research. Also the role of inter-chain frustration $J_{\times}$promises to be an interesting topic in the future.

\section{ACKNOWLEDGMENTS}

We thank Masaki Oshikawa, Wei Li, and Zhidan Wang for the useful discussion and/or critical reading of the manuscript. This research was supported by the Nachwuchsring of the TU Kaiserslautern, by the German Research Foundation (DFG) via the Collaborative Research Centers SFB/TR49 and SFB/TR185 (OSCAR), by the the National Natural Science Foundation of China (Grants No. 11474029 and 11574200), and by the National Program on Key Research Project (Grants No. 2016YFA0300501). We gratefully acknowledge the computing time granted by the John von Neumann Institute for Computing (NIC) and provided on the supercomputer JURECA at Jülich Supercomputing Centre (JSC). We also thank the computational resources provided by Physics Laboratory for High Performance Computing (RUC), and by Shanghai Supercomputer Center where most of the computations are carried out. 


\section{Appendix A: Bosonization}

In this appendix we provide additional information for the field theory derivation and the corresponding renormalization group (RG) flow.

For the derivation of the bosonization formulas and the operator product expansion (OPE) it is useful to consider an interacting spinor Fermion model as the underlying physical realization where only the spin channel will be considered in the low-energy limit $\underline{\underline{3}}$ For the half-filled Hubbard model, the charge channel is gapped and the Heisenberg couplings considered in the paper correspond to the spin channel.

The spin currents are expressed as in terms of chiral fermionic field operators

$$
J_{\kappa}^{a}\left(z_{\kappa}\right)=: \psi_{\kappa \eta}^{\dagger} \frac{\sigma_{\eta \eta^{\prime}}^{a}}{2} \psi_{\kappa \eta^{\prime}}:\left(z_{\kappa}\right)
$$

where $\sigma^{a}$ are the Pauli matrices, the sum over spin components $\eta=\uparrow, \downarrow$ is implied, and $\kappa$ denotes the chirality ( $\kappa=R / L$ or $\kappa=+/-$ respectively). The chiral complex coordinates are $z_{\kappa}=-\kappa i x+v \tau$. The dimerization and staggered magnetization operators are given by ${ }^{88}$

$$
\begin{aligned}
\epsilon(z) & \sim \frac{i}{2}\left[: \psi_{R \eta}^{\dagger} \psi_{L \eta}:(z)-: \psi_{L \eta}^{\dagger} \psi_{R \eta}:(z)\right], \\
n^{a}(z) & \sim \frac{1}{2} \sigma_{\eta \eta^{\prime}}^{a}\left[: \psi_{R \eta}^{\dagger} \psi_{L \eta^{\prime}}:(z)+: \psi_{L \eta}^{\dagger} \psi_{R \eta^{\prime}}:(z)\right],
\end{aligned}
$$

where $z$ implies a dependence on both chiral variables $z_{L}$, $z_{R}$.

The OPEs between $J_{\kappa}^{a}, \epsilon$, and $n^{a}$ can be calculated using Wick's theorem 66 and the two-point correlation function

$$
\left\langle\psi_{\kappa \eta}\left(z_{\kappa}\right) \psi_{\kappa^{\prime} \eta^{\prime}}^{\dagger}\left(w_{\kappa^{\prime}}\right)\right\rangle=\delta_{\kappa \kappa^{\prime}} \delta_{\eta \eta^{\prime}} \frac{\gamma}{z_{\kappa}-w_{\kappa}},
$$

where $\gamma$ depends on the chosen normalization. Here $\gamma=$ $1 / 2 \pi$. The required fundamental OPEs are ${ }^{88}$

$$
\begin{aligned}
J_{\kappa}^{a}\left(z_{\kappa}\right) \epsilon(w) & =i \kappa \frac{\gamma / 2}{z_{\kappa}-w_{\kappa}} n^{a}(w), \\
J_{\kappa}^{a}\left(z_{\kappa}\right) n^{b}(w) & =i \frac{\gamma / 2}{z_{\kappa}-w_{\kappa}}\left[\epsilon_{a b c} n^{c}(w)-\kappa \delta_{a b} \epsilon(w)\right], \\
J_{\kappa}^{a}\left(z_{\kappa}\right) J_{\kappa^{\prime}}^{b}\left(w_{\kappa^{\prime}}\right) & =\delta_{\kappa \kappa^{\prime}}\left[\frac{\left(\gamma^{2} / 2\right) \delta_{a b}}{\left(z_{\kappa}-w_{\kappa}\right)^{2}}+i \epsilon_{a b c} \gamma \frac{J_{\kappa}^{c}\left(w_{\kappa}\right)}{z_{\kappa}-w_{\kappa}}\right], \\
\epsilon(z) \epsilon(w) & =\frac{\gamma^{2}}{|z-w|}-|z-w| \mathbf{J}_{R} \cdot \mathbf{J}_{L}(w) \\
n^{a}(z) \epsilon(w) & =-i \gamma|z-w|\left[\frac{J_{R}^{a}\left(w_{R}\right)}{z_{L}-w_{L}}-\frac{J_{L}^{a}\left(w_{L}\right)}{z_{R}-w_{R}}\right], \\
n^{a}(z) n^{b}(w) & =|z-w|\left[\hat{Q}^{a b}(w)+\frac{\gamma^{2} \delta_{a b}}{\mid z-w^{2}}+i \epsilon_{a b c} \gamma\right. \\
& \left.\times\left[\frac{J_{R}^{c}\left(w_{R}\right)}{z_{L}-w_{L}}+\frac{J_{L}^{c}\left(w_{L}\right)}{z_{R}-w_{R}}\right]\right]
\end{aligned}
$$

where $\delta_{a b}$ is the Kronecker $\delta$-function, and $\epsilon_{a b c}$ the Levi Civita symbol. $Q^{a b}$ denotes the zeroth-order contraction (a)

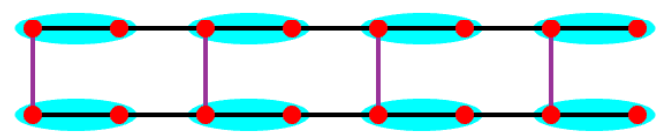

(b)

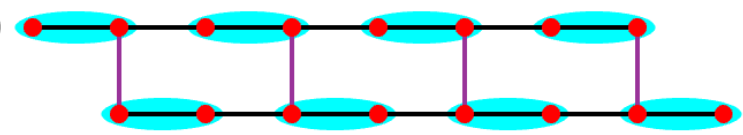

FIG. 16. (a) Configuration of OBC with the corresponding possible columnar pattern of singlet dimers (cyan ellipsiods). (b) Shifted OBC with a staggered dimer pattern.

between the fermionic fields,

$$
\hat{Q}^{a b}=\frac{1}{2} \sigma_{\eta \eta^{\prime}}^{a} \sigma_{\tau \tau^{\prime}}^{b} \psi_{R \eta}^{\dagger} \psi_{L \eta^{\prime}} \psi_{L \tau}^{\dagger} \psi_{R \tau^{\prime}}
$$

As it turns out only the trace of this operator is relevant for the RG flow of spin ladders, which reads

$$
\hat{Q}^{a a}=\mathbf{J}_{R} \cdot \mathbf{J}_{L}
$$

after freezing out charge degrees of freedom.

The evolution of the bare couplings, is determined from the OPEs of the perturbing operators, using 69

$$
\frac{d \lambda_{k}}{d l}=\left(2-d_{k}\right) \lambda_{k}-\frac{\pi}{v} \sum_{i, j} C_{i j k} \lambda_{i} \lambda_{j}
$$

where $d_{k}$ is the scaling dimension of the operator, $v$ the velocity, and $C_{i j k}$ the coefficient extracted from the OPE

$$
O_{i}(z, \bar{z}) O_{j}(w, \bar{w}) \sim \sum_{k} C_{i j k} \frac{O_{k}}{(z-w)^{\nu_{k}}(\bar{z}-\bar{w})^{\bar{\nu}_{k}}}
$$

with $\nu, \bar{\nu}$ the holomorphic and antiholomorphic conformal dimension. Using Eqs. (A4) and A7) we compute the corresponding RG flow.

For the lower part of the phase diagram in Fig. 3 . where $J_{1} \gg J_{2}, J_{\perp}$ is assumed, the operators content in Eq. (7) can be obtained. Using the fundamental OPEs in Eq. (A4) we arrive at the RG equations in Eq. (10).

In the opposite regime, where the coupling $J_{2}$ is the strongest interaction in the system, $J_{2} \gg J_{1}, J_{\perp}$, the lattice Hamiltonian can be written as

$$
\begin{aligned}
H & =J_{2} \sum_{\bar{\alpha}=1}^{4} \sum_{j} \mathbf{S}_{j, \bar{\alpha}} \cdot \mathbf{S}_{j+1, \bar{\alpha}}+J_{\perp} \sum_{j} \mathbf{S}_{j, 2} \cdot \mathbf{S}_{j, 4} \\
& +J_{1} \sum_{\bar{\alpha}=1}^{2} \sum_{j} \mathbf{S}_{j, 2 \bar{\alpha}-1} \cdot\left(\mathbf{S}_{j, 2 \bar{\alpha}}+\mathbf{S}_{j+1,2 \bar{\alpha}}\right) .
\end{aligned}
$$

Bosonizing this Hamiltonian gives the operators in Eq. (12). The RG equations in Eq. (14) are derived from the OPEs in Eq. (A4). 

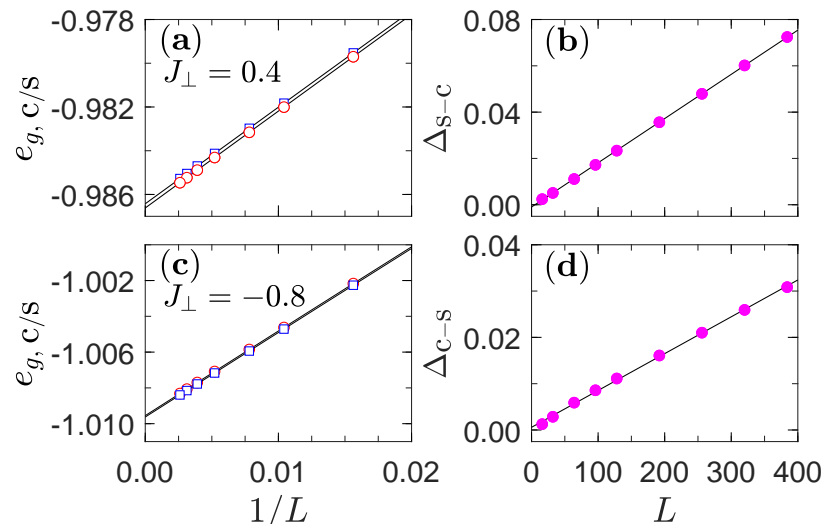

FIG. 17. (a) Ground state energy per-rung $e_{g}=E_{g} / L$ for $J_{\perp}=0.4$ and $J_{2}=1.0$ with $\mathrm{OBC}$ and shifted OBC. (b) corresponding total energy difference $\Delta_{\mathrm{S}-\mathrm{C}}=E_{g}^{\mathrm{S}}-E_{g}^{\mathrm{C}}$. Analogous results for $J_{\perp}=-0.8$ and $J_{2}=1.0$ are shown in (c) and (d).

\section{Appendix B: Compatible boundary conditions}

The CD and SD phases are both doubly degenerate for $\mathrm{PBC}$, since there are two equivalent dimer patterns related by symmetry (reflection and translation). For $\mathrm{OBC}$, the system will choose the lower energy state with more strong bonds, so the geometry at edges lifts the degeneracy and fixes the dimer pattern. In particular, the edges may also affect the relative energy of the SD and CD patterns. We therefore identify two suitable boundary conditions, which are compatible with either the SD or the CD pattern respectively, as shown in Fig. 16. This allows to always identify the lowest possible energetic configuration in the simulations.

For the columnar arrangement we use ordinary OBC with an even number of rungs as shown in Fig. 16)(a), which prefers the CD phase. In the staggered pattern, there is a relative shift of the dimerization by one site on the two legs, so we accordingly also shift the OBC (shifted OBC) as shown in Fig. 16(b), which prefers the $\mathrm{SD}$ phase. For shifted OBC there is an odd number of rungs but the first and last rung only contain one spin, so the number of spins remains divisible by 4 . As an example, we now focus on the ground state energies for different boundary conditions at $J_{\perp}=0.4$ and -0.8 with $J_{2}=1.0$ in Fig. 17. The corresponding ground-state energies for both $\mathrm{OBC}$ and shifted $\mathrm{OBC}$ are shown in Fig. 17. As shown in Fig. 17(a), for $J_{\perp}=0.4$ the groundstate energies per-rung $e_{g, \mathrm{c}}=-0.986607(8) J_{1}$ for $\mathrm{OBC}$ and $e_{g, \mathrm{~s}}=-0.986418(6) J_{1}$ for shifted OBC, have a small energy difference of $1.9(1) \times 10^{-4}$, independent of $L$. The total energy difference $\Delta_{\mathrm{S}-\mathrm{C}}$ therefore increases with $L$ with the corresponding slope shown in Fig. 17(b). This implies that the bulk energy is affected by the boundaries, so the correlations of the ground states are different throughout the system (in this case CD and SD). Therefore the correct CD pattern can only be obtained with the corresponding compatible OBC. Fig 17(c,d) show the analogous behavior on the ferromagnetic side $J_{\perp}=-0.8$ where shifted OBC must be used to identify the correct ground state correlations (SD phase).
* shijiehu@gmail.com

$\dagger$ xiaoqunwang@sjtu.edu.cn

1 H. T. Diep, ed., Frustrated spin systems. Singapore: World Scientific, 2005.

2 X.-F. Zhang, S. Hu, A. Pelster, and S. Eggert, Quantum domain walls induce incommensurate supersolid phase on the anisotropic triangular lattice, Phys. Rev. Lett. 117, 193201 (2016).

3 I. Affleck, Field theory methods and quantum critical phenomena in Fields, strings and critical phenomena (E. Brézin and J. Zinn-Justin, eds.), 563, North-Holland, Amsterdam, 1990. (proceedings of Les Houches Summer School, 1988).

${ }^{4}$ K. Okamoto and K. Nomura, Fluid-dimer critical point in $S=1 / 2$ antiferromagnetic Heisenberg chain with next nearest neighbor interactions, Phys. Lett. A 169, 433 (1992).

5 G. Castilla, S. Chakravarty, and V. J. Emery, Quantum magnetism of $\mathrm{CuGeO}_{3}$, Phys. Rev. Lett. 75, 1823 (1995).

6 S. Eggert, Numerical evidence for multiplicative logarithmic corrections from marginal operators, Phys. Rev. B 54, R9612 (1996).

7 S. R. White and I. Affleck, Dimerization and incommensurate spiral spin correlations in the zigzag spin chain: Analogies to the Kondo lattice, Phys. Rev. B 54, 9862 (1996).

8 C. K. Majumdar and D. K. Ghosh, On next-nearest- neighbor interaction in linear chain. I, J. Math. Phys. 10, 1388 (1969).

${ }^{9}$ C. K. Majumdar and D. K. Ghosh, On next-nearestneighbor interaction in linear chain. II, J. Math. Phys. 10, 1399 (1969).

10 A. Deschner and E. S. Sørensen, Incommensurability effects in odd length $J_{1}-J_{2}$ quantum spin chains: On-site magnetization and entanglement, Phys. Rev. B 87, 094415 (2013).

11 F. Haldane, Continuum dynamics of the 1-D Heisenberg antiferromagnet: Identification with the $\mathrm{O}(3)$ nonlinear sigma model, Phys. Lett. A 93, 464 (1983).

12 F. D. M. Haldane, Nonlinear field theory of largespin Heisenberg antiferromagnets: semiclassically quantized solitons of the one-dimensional easy-axis Néel state, Phys. Rev. Lett. 50, 1153 (1983).

13 M. den Nijs and K. Rommelse, Preroughening transitions in crystal surfaces and valence-bond phases in quantum spin chains, Phys. Rev. B 40, 4709 (1989).

14 T. Kennedy and H. Tasaki, Hidden $Z_{2}-Z_{2}$ symmetry breaking in Haldane-gap antiferromagnets, Phys. Rev. B 45, 304 (1992).

15 A. Kolezhuk, R. Roth, and U. Schollwöck, First order transition in the frustrated antiferromagnetic Heisenberg $S=1$ quantum spin chain, Phys. Rev. Lett. 77, 5142 (1996).

16 A. Kolezhuk, R. Roth, and U. Schollwöck, Variational and 
density-matrix renormalization-group studies of the frustrated antiferromagnetic Heisenberg $S=1$ quantum spin chain, Phys. Rev. B 55, 8928 (1997).

17 T. Hikihara, M. Kaburagi, H. Kawamura, and T. Tonegawa, Ground state phase diagram of frustrated $S=1$ XXZ chains: chiral ordered phases, J. Phys. Soc. Jpn. 69, 259 (2000).

18 A. K. Kolezhuk and U. Schollwöck, Connectivity transition in the frustrated $S=1$ chain revisited, Phys. Rev. B 65, 100401 (2002).

19 J. H. Pixley, A. Shashi, and A. H. Nevidomskyy, Frustration and multicriticality in the antiferromagnetic spin-1 chain, Phys. Rev. B 90, 214426 (2014).

20 N. Chepiga, I. Affleck, and F. Mila, Dimerization transitions in spin-1 chains, Phys. Rev. B 93, 241108 (2016).

${ }^{21}$ N. Chepiga, I. Affleck, and F. Mila, Comment on "Frustration and multicriticality in the antiferromagnetic spin-1 chain", Phys. Rev. B 94, 136401 (2016).

22 N. Chepiga, I. Affleck, and F. Mila, Spontaneous dimerization, critical lines, and short-range correlations in a frustrated spin-1 chain, Phys. Rev. B 94, 205112 (2016).

23 E. Dagotto, J. Riera, and D. Scalapino, Superconductivity in ladders and coupled planes, Phys. Rev. B 45, 5744 (1992).

24 T. Barnes, E. Dagotto, J. Riera, and E. S. Swanson, Excitation spectrum of Heisenberg spin ladders, Phys. Rev. B 47, 3196 (1993).

25 S. R. White, R. M. Noack, and D. J. Scalapino, Resonating valence bond theory of coupled Heisenberg chains, Phys. Rev. Lett. 73, 886 (1994).

26 M. Azzouz, L. Chen, and S. Moukouri, Calculation of the singlet-triplet gap of the antiferromagnetic Heisenberg model on a ladder, Phys. Rev. B 50, 6233 (1994).

27 E. Dagotto and T. M. Rice, Surprises on the way from oneto two-dimensional quantum magnets: the ladder materials, Science 271, 618 (1996).

28 K. P. Schmidt, H. Monien, and G. S. Uhrig, Rung-singlet phase of the $S=1 / 2$ two-leg spin-ladder with four-spin cyclic exchange, Phys. Rev. B 67, 184413 (2003).

${ }^{29}$ O. A. Starykh and L. Balents, Dimerized phase and transitions in a spatially anisotropic square lattice antiferromagnet, Phys. Rev. Lett. 93, 127202 (2004).

30 H.-H. Hung, C.-D. Gong, Y.-C. Chen, and M.-F. Yang, Search for quantum dimer phases and transitions in a frustrated spin ladder, Phys. Rev. B 73, 224433 (2006).

31 E. H. Kim, Ö. Legeza, and J. Sólyom, Topological order, dimerization, and spinon deconfinement in frustrated spin ladders, Phys. Rev. B 77, 205121 (2008).

${ }^{32}$ T. Hikihara and O. A. Starykh, Phase diagram of the frustrated spin ladder, Phys. Rev. B 81, 064432 (2010).

33 G. Barcza, Ö. Legeza, R. M. Noack, and J. Sólyom, Dimerized phase in the cross-coupled antiferromagnetic spin ladder, Phys. Rev. B 86, 075133 (2012).

34 T. Vekua and A. Honecker, Quantum dimer phases in a frustrated spin ladder: Effective field theory approach and exact diagonalization, Phys. Rev. B 73, 214427 (2006).

35 G.-H. Liu, H.-L. Wang, and G.-S. Tian, Existence of dimerized phases in frustrated spin ladder models, Phys. Rev. B 77, 214418 (2008).

36 A. Lavarélo, G. Roux, and N. Laflorencie, Melting of a frustration-induced dimer crystal and incommensurability in the $J_{1}-J_{2}$ two-leg ladder, Phys. Rev. B 84, 144407 (2011).
37 Y.-C. Li and H.-Q. Lin, Quantum phase diagram of the frustrated spin ladder with next-nearest-neighbor interactions, New J. Phys. 14, 063019 (2012).

38 G.-H. Liu, X.-Y. Deng, and R. Wen, Phase diagram of the frustrated spin ladder with the next nearest intrachain couplings, Physica B: Cond. Mat. 407, 2068 (2012).

39 T. Sugimoto, M. Mori, T. Tohyama, and S. Maekawa, Effects of frustration on magnetic excitations in a two-leg spin-ladder system, Phys. Rev. B 87, 155143 (2013).

40 A. Metavitsiadis, D. Sellmann, and S. Eggert, Spin-liquid versus dimer phases in an anisotropic $J_{1}-J_{2}$ frustrated square antiferromagnet, Phys. Rev. B 89, 241104 (2014).

41 A. Metavitsiadis and S. Eggert, Competing phases in spin ladders with ring exchange and frustration, Phys. Rev. B 95, 144415 (2017).

${ }^{42}$ Y. Xian, Rigorous results on a first-order phase transition in antiferromagnetic spin- $1 / 2$ coupled chains, Phys. Rev. B 52, 12485 (1995).

43 X. Wang, Low energy Properties of a frustrated antiferromagnetic spin-1/2 ladder, Mod. Phys. Lett. B 14, 327 (2000).

44 F. Amiri, G. Sun, H.-J. Mikeska, and T. Vekua, Groundstate phases of a rung-alternated spin-1/2 Heisenberg ladder, Phys. Rev. B 92, 184421 (2015).

45 A. Mattsson, P. Fröjdh, and T. Einarsson, Frustrated honeycomb Heisenberg antiferromagnet: A Schwinger-boson approach, Phys. Rev. B 49, 3997 (1994).

46 J. Oitmaa and R. R. P. Singh, Phase diagram of the $J_{1}-$ $J_{2}-J_{3}$ Heisenberg model on the honeycomb lattice: A series expansion study, Phys. Rev. B 84, 094424 (2011).

47 J. Reuther, D. A. Abanin, and R. Thomale, Magnetic order and paramagnetic phases in the quantum $J_{1}-J_{2}-J_{3}$ honeycomb model, Phys. Rev. B 84, 014417 (2011).

${ }^{48}$ P. H. Y. Li, R. F. Bishop, D. J. J. Farnell, and C. E. Campbell, Phase diagram of a frustrated Heisenberg antiferromagnet on the honeycomb lattice: The $J_{1}-J_{2}-J_{3}$ model, Phys. Rev. B 86, 144404 (2012).

${ }^{49}$ F. Ferrari, S. Bieri, and F. Becca, Competition between spin liquids and valence-bond order in the frustrated spin$1 / 2$ Heisenberg model on the honeycomb lattice, Phys. Rev. B 96, 104401 (2017).

50 Y.-Y. Zhu, C. Cui, N. Li, B.-W. Wang, Z.-M. Wang, and $\mathrm{S}$. Gao, Constructing a series of azide-bridged $\mathrm{Cu}^{\mathrm{II}}$ magnetic low-dimensional coordination polymers by using pybox ligands, Eur. J. Inorg. Chem. 2013, 3101 (2013).

51 F. Görg, M. Messer, K. Sandholzer, G. Jotzu, R. Desbuquois, and T. Esslinger, Enhancement and sign change of magnetic correlations in a driven quantum many-body system, Nature 553, 481 (2018).

52 S. R. White, Density matrix formulation for quantum renormalization groups, Phys. Rev. Lett. 69, 2863 (1992).

53 S. R. White, Density-matrix algorithms for quantum renormalization groups, Phys. Rev. B 48, 10345 (1993).

54 I. Peschel, X. Q. Wang, M. Kaulke, and K. Hallberg, Density-matrix renormalization. Springer Berlin Heidelberg, 1999.

55 U. Schollwöck, The density-matrix renormalization group, Rev. Mod. Phys. 77, 259 (2005).

56 I. P. McCulloch, Infinite size density matrix renormalization group, preprint arXiv:0804.2509 (unpublished).

57 S. Hu, B. Normand, X. Wang, and L. Yu, Accurate determination of the Gaussian transition in spin-1 chains with single-ion anisotropy, Phys. Rev. B 84, 220402 (2011).

58 S. Hu, A. M. Turner, K. Penc, and F. Pollmann, Berry- 
phase-induced dimerization in one-dimensional quadrupolar systems, Phys. Rev. Lett. 113, 027202 (2014).

${ }^{59}$ K. Hida, Ground-state phases of anisotropic mixed diamond chains with spins 1 and 1/2, J. Phys. Soc. Jpn. 83, 114711 (2014).

60 J. A. Kjäll, M. P. Zaletel, R. S. K. Mong, J. H. Bardarson, and F. Pollmann, Phase diagram of the anisotropic spin-2 XXZ model: Infinite-system density matrix renormalization group study, Phys. Rev. B 87, 235106 (2013).

${ }^{61}$ F. Pollmann, E. Berg, A. M. Turner, and M. Oshikawa, Symmetry protection of topological phases in onedimensional quantum spin systems, Phys. Rev. B 85, 075125 (2012).

${ }^{62}$ F. Pollmann, A. M. Turner, E. Berg, and M. Oshikawa, Entanglement spectrum of a topological phase in one dimension, Phys. Rev. B 81, 064439 (2010).

63 S. Eggert and I. Affleck, Magnetic impurities in half-integer-spin Heisenberg antiferromagnetic chains, Phys. Rev. B 46, 10866 (1992).

${ }^{64}$ I. Affleck, Exact critical exponents for quantum spin chains, non-linear-models and the quantum hall effect, Nucl. Phys. B 265, 409 (1986).

65 M. A. Tsvelik, Quantum field theory in condensed matter physics. Cambridge university press, 2003.

66 P. Di Francesco, P. Mathieu, and D. Senechal, Conformal field theory. Springer, 1997.

${ }^{67}$ I. Affleck, T. Kennedy, E. H. Lieb, and H. Tasaki, Valence bond ground states in isotropic quantum antiferromagnets, Comm. Math. Phys. 115, 477 (1988).

68 S. Eggert and I. Affleck, Magnetic impurities in half-integer-spin Heisenberg antiferromagnetic chains, Phys. Rev. B 46, 10866 (1992).

69 J. Cardy, Scaling and renormalization in statistical physics. Cambridge university press, 1996.

70 P. Di Francesco, P. Mathieu, and D. Senechal, Conformal field theory. Springer, 1997.

71 M. A. Tsvelik, Quantum field theory in condensed matter physics. Cambridge university press, 2003.

72 D. Sénéchal, An introduction to bosonization CRM series in mathematical Physics, ch. 4, 139, Springer, 2004.

73 F. Pollmann and A. M. Turner, Detection of symmetryprotected topological phases in one dimension, Phys. Rev. B 86, 125441 (2012).

74 L. Campos Venuti, C. Degli Esposti Boschi, M. Roncaglia, and A. Scaramucci, Local measures of entanglement and critical exponents at quantum phase transitions, Phys. Rev. A 73, 010303 (2006).

75 A. Osterloh, L. Amico, G. Falci, and R. Fazio, Scaling of entanglement close to a quantum phase transition, Nature 416, 608 (2002).

76 L.-A. Wu, M. S. Sarandy, and D. A. Lidar, Quantum phase transitions and bipartite entanglement, Phys. Rev. Lett. 93, 250404 (2004).

77 L. Tagliacozzo, T. R. de Oliveira, S. Iblisdir, and J. I. Latorre, Scaling of entanglement support for matrix product states, Phys. Rev. B 78, 024410 (2008).

78 J. I. Latorre and A. Riera, A short review on entanglement in quantum spin systems, J. Phys. A: Math. Theo. 42, 504002 (2009).

79 N. Laflorencie, Quantum entanglement in condensed matter systems, Phys. Rep. 646, 1 (2016).

$80 \mathrm{X}$. Wang, Boundary and impurity effects on the entanglement of Heisenberg chains, Phys. Rev. E 69, 066118 (2004).

81 N. Laflorencie, E. S. Sørensen, M.-S. Chang, and I. Affleck, Boundary effects in the critical scaling of entanglement entropy in 1D systems, Phys. Rev. Lett. 96, 100603 (2006).

82 H. F. Song, S. Rachel, and K. Le Hur, General relation between entanglement and fluctuations in one dimension, Phys. Rev. B 82, 012405 (2010).

83 H. F. Song, S. Rachel, C. Flindt, I. Klich, N. Laflorencie, and K. Le Hur, Bipartite fluctuations as a probe of manybody entanglement, Phys. Rev. B 85, 035409 (2012).

${ }^{84} \mathrm{H}$. Li and F. D. M. Haldane, Entanglement spectrum as a generalization of entanglement entropy: identification of topological order in non-Abelian fractional quantum Hall effect states, Phys. Rev. Lett. 101, 010504 (2008).

85 E. S. Sorensen, S. Eggert, and I. Affleck, Integrable versus non-integrable spin chain impurity models, J. Phys. A: Math. Gen. 26, 6757 (1993).

86 M. Reigrotzki, H. Tsunetsugu, and T. M. Rice, Strongcoupling expansions for antiferromagnetic Heisenberg spinone-half ladders, J. Phys: Condens. Matter 6, 9235 (1994).

87 D. C. Cabra, A. Honecker, and P. Pujol, Magnetization curves of antiferromagnetic Heisenberg spin-1/2 ladders, Phys. Rev. Lett. 79, 5126 (1997).

88 O. A. Starykh, A. Furusaki, and L. Balents, Anisotropic pyrochlores and the global phase diagram of the checkerboard antiferromagnet, Phys. Rev. B 72, 094416 (2005). 